\title{
Classification of physical activities and sedentary behavior using raw data of 3D hip acceleration
}

\author{
P. Tjurin ${ }^{1}$, M. Niemelä ${ }^{1}$, M. Huusko ${ }^{1}$, R. Ahola ${ }^{2}$, M. Kangas ${ }^{1,3}$ and T. Jämsä ${ }^{1,3,4}$ \\ ${ }^{1}$ Research Unit of Medical Imaging, Physics and Technology (MIPT), University of Oulu, Oulu, Finland \\ ${ }_{2}$ Polar Electro Oy, Kempele, Finland \\ ${ }^{3}$ Medical Research Center, University of Oulu and Oulu University Hospital, Oulu, Finland \\ ${ }^{4}$ Department of Diagnostic Radiology, Oulu University Hospital, Oulu, Finland
}

\begin{abstract}
The purpose of this study was to develop and validate an algorithm for classifying physical activity (PA) classes and sedentary behavior (SED) from raw acceleration signal measured from hip. Twenty-two adult volunteers completed a pre-defined set of controlled and supervised activities. The activities included nine daily PAs. The participants performed PA trials while wearing a hip-worn 3D accelerometer. Indirect calorimetry was used for measuring energy expenditure. The raw acceleration data were used for training and testing a prediction model in MATLAB environment. The prediction model was built using bagged trees classifier and the most suitable extracted features (mean, maximum, minimum, zero crossing rate, and mean amplitude deviation) were selected using a sequential forward selection method. Leave-one-out cross validation was used for validation. Activities were classified as lying, sitting, light PA (standing, table wiping, floor cleaning, slow walking), moderate PA (fast walking) and vigorous PA (soccer and jogging). The oxygen consumption data were used for estimating the intensity of measured PA. Total accuracy of the prediction model was $96.5 \%$. Mean sensitivity of the model was 95.5\% (SD 3.5) and mean specificity 99.1\% (SD 0.5). Based on the results PA types can be classified from raw data of the hipworn 3D accelerometer using supervised machine learning techniques with a high sensitivity and specificity. The developed algorithm has a potential for objective evaluations of PA and SED.
\end{abstract}

Keywords - physical activity, sedentary behavior, accelerometer, machine learning, raw data

\section{INTRODUCTION}

Physical activity (PA) enhances health outcomes by reducing the risk of morbidity and mortality [1]. On the other hand, excessive time spent in sedentary behavior (SED) and especially prolonged periods of sitting are independent risk factors for chronic diseases despite the amount of PA [2-3]. Therefore, objective methods to measure PA and the patterns of SED are required to improve understanding of their health effects.

There are several types of PA monitors and analysis methods available [4]. The output of different monitors is dependent on the used signal processing methods and the thresholds of PA levels. As a result, different types of accelerometers are not interchangeable with each other when measuring PA in free-living conditions. [5] Researchers have proposed the use of raw acceleration signal instead of some specific unit e.g. counts [6]. However, there is still missing consensus of the accurate analysis technique [4, 6-7].

Mean amplitude deviation (MAD) has been suggested a potential universal feature for analyzing raw data of a 3D accelerometer [8]. MAD indicates the mean difference of the data samples from the mean acceleration and it is computed from the magnitude vector of three orthogonal acceleration signals.

Machine learning (ML) techniques for PA classification have shown promising results [9-11]. Compared to the traditional processing methods the advantage of $\mathrm{ML}$ is that it can extract nonlinearities and complex dependencies from acceleration signals. Using ML methods PA classification can be done without specific thresholds e.g. METs or counts. [10] The aim of this study was to develop and validate an algorithm for classifying PA based on intensity and SED from raw acceleration data using MAD and ML techniques.

\section{MethodS}

\section{A. Participants}

Twenty-two 17-58 years old healthy volunteers were recruited for measurements. All participants were asked to provide written consent to take part in the study. Oral and written information about the study were given to the participants before obtaining the consent. The participants had right to refuse to participate in or to withdraw from the study. The study followed the ethical principles for medical research involving human subjects in Finland and the Declaration of Helsinki.

The participants were guided to abstain from alcohol and unaccustomed hard activity or exercise for 24 hours prior to measurements. Gender, age, and long-term PA level of the participants were assessed by a questionnaire. Training background were collected using a six-point scale $(0-1,1-3,3-5$, $5-8,8-12$, and $>12 \mathrm{~h} /$ week) by asking to choose the level that 
best describes the overall amount and intensity of their PA during the past three months.

In addition, height and body mass of the participants were measured prior to PA measurements. Height was measured to the nearest $0.1 \mathrm{~cm}$ using a stadiometer and body mass was measured to the nearest $0.1 \mathrm{~kg}$ using a digital scale. Body mass index (BMI) was calculated using the measured height and weight data $\left(\mathrm{kg} / \mathrm{m}^{2}\right)$. The demographic characteristics of the participants are shown in Table 1.

Table 1 Demographic characteristics of the study participants

\begin{tabular}{lccc}
\hline & Women $(\mathrm{n}=11)$ & Men $(\mathrm{n}=11)$ & Total $(\mathrm{n}=22)$ \\
\hline Age (years) & $26.0(11.3)$ & $29.0(11.6)$ & $27.5(11.2)$ \\
Height $(\mathrm{cm})$ & $165.8(3.2)$ & $180.1(3.5)$ & $173.0(8.0)$ \\
Body mass $(\mathrm{kg})$ & $66.8(8.2)$ & $84.5(6.7)$ & $75.7(11.7)$ \\
BMI $\left(\mathrm{kg} / \mathrm{m}^{2}\right)$ & $24.2(2.4)$ & $26.0(1.6)$ & $25.1(2.2)$ \\
\hline
\end{tabular}

Data are mean or median (SD).

\section{B. Data set}

Volunteers completed a pre-defined set of controlled and supervised activities for four minutes each, with a $0.5-4 \mathrm{mi}-$ nute resting period between activities depending on the intensity of the previous activity. The protocol included a set of nine daily activities with increasing intensity (hanging out on a sofa, sitting at a computer, standing/poster viewing, wiping and setting up kitchen table, floor cleaning, slow walking, fast walking, soccer passing, and jogging). The selected activities represented typical free-living activities and ranged in intensity from sedentary to vigorous [12]. The participants performed walking and jogging at self-selected speed. Fast walking, soccer, and jogging was conducted on an outdoor track. The participants were guided orally during the measurements. Before the beginning of a new activity the participants were asked to stand still.

Triaxial accelerometer (Hookie AM20, Traxmeet Ltd, Espoo, Finland) was attached to the participant's right hip on the elastic belt. Acceleration data were collected in a raw mode at $100 \mathrm{~Hz}$ in range $\pm 16 \mathrm{~g}$. The manufacturer's default values for thresholds of acceleration sensor were used.

Oxygen consumption was measured with indirect calorimetry (COSMED K4 b2, Cosmed Ltd, Rome, Italy) and used as a criterion measure. COSMED K4 b2 has been shown to reliably measure oxygen consumption [13]. Before each measurement and transition to the outdoor track, indirect calorimetry was calibrated. Indirect calorimetry was worn as a vest with a rubber facemask (Hans Rudolph, Kansas City, USA) and the participants were not allowed to speak during activities. Because wearing the indirect calorimetry produced variability in hanging out on a sofa, six participants performed lying on a sofa without oxygen consumption measurement.

The acceleration data from the nine different daily activities were used for training and testing a prediction model. The complete data processing and training of the classifier were conducted using MATLAB R2016b (The MathWorks, Inc).

\section{Data analyses}

The raw acceleration data were transformed into g-units. The signal magnitude vector (SMV) was calculated from three-dimensional acceleration data using the following equation:

$$
S M V=\sqrt{a c c_{-} x^{2}+a c c_{-} y^{2}+a c c_{-} z^{2}}
$$

The acceleration signals were filtered with $4^{\text {th }}$ order Butterworth lowpass filter with cut off frequency of $20 \mathrm{~Hz}$. Human movement is typically under $20 \mathrm{~Hz}$ so a high frequency noise should be filtered [14]. An influence of ground reaction force was removed from the SMV by filtering the signal with $4^{\text {th }}$ order Butterworth highpass filter with cut off frequency of $0.5 \mathrm{~Hz}$. The acceleration signals were resampled from 100 $\mathrm{Hz}$ to $10 \mathrm{~Hz}$ for a lighter performance. A high accuracy for classification of typical human PAs could be achieved using data sampled at $10 \mathrm{~Hz}$ [15-16].

Classification was conducted using supervised ML techniques. In order to extract features the acceleration data were windowed. Window size was set to $5 \mathrm{~s}$ (50 data samples) and the slide between two adjacent windows was 2.5 seconds ( 25 data samples). Window size of approximately $5 \mathrm{~s}$ has been shown to achieve sufficient accuracy in detecting physical activity intensities [17]. For reducing the number of misclassified windows, the final classification was performed based on the classification results of six adjacent windows, i.e. an activity had to stay same for $30 \mathrm{~s}$ in order to be classified as a new activity.

Classification features were extracted from three dimensional acceleration data and SMV. The extracted features were mean, MAD, zero crossing rate (ZCR), minimum, maximum, and difference between maximum and minimum so that a total number of features was 21. A sequential forward selection method (SFS) was employed for a selection of the most suitable features.

The selected features (mean, minimum, maximum, ZCR, and MAD) were used for training a classification model. In order to find the most applicable classifier experiments were performed using several standard ML classifiers: decision trees (boosted and bagged), k nearest neighbors ( $\mathrm{kNN}$ ), support vector machines (SVM), and linear discriminant analysis (LDA). Leave-one-out cross validation was employed for validation of the prediction models in order to obtain user- 
independent results. In the leave-one-out method the data of each participant was used in turn as a test data and the remaining data were used for model training.

Total accuracy for each classifier was obtained by dividing the number of the correct predictions by the total number of the predictions, see Table 2. The bootstrap aggregated (bagged) trees classifier provided the best results and was chosen to be used in the final classification process. In bagged trees classifier several decision trees were combined to reduce variance of the prediction [18]. The classifier trained multiple decision tree models on different randomly selected bootstrap samples of the data and generated an estimate by averaging the classification results of the models.

Table 2 Total accuracies of the classifiers

\begin{tabular}{lc}
\hline Classifier & Total accuracy \\
\hline Bagged trees & $96.5 \%$ \\
Boosted trees & $96.3 \%$ \\
kNN & $90.7 \%$ \\
SVM & $89.0 \%$ \\
LDA & $84.6 \%$ \\
\hline
\end{tabular}

Mean value of metabolic equivalent (MET) for each activity was calculated from the data collected with indirect calorimetry. Breath-by-breath $\mathrm{VO}_{2}$ data of 12 participants was filtered with a $15 \mathrm{~s}$ average filter using the $\mathrm{K} 4 \mathrm{~b} 2$ software. Conversion from $\mathrm{VO}_{2}$ data to METs was computed using the standard convention of $1 \mathrm{MET}=3.5 \mathrm{ml} \mathrm{O} / \mathrm{min} / \mathrm{kg}$. The mean MET values of each activity were calculated from steady state. PA classification and METs of activities are shown in Table 3.

Table 3 PA classification and METs

\begin{tabular}{llcc}
\hline & & \multicolumn{2}{c}{ METs } \\
\cline { 3 - 4 } PA classification & Activity & Mean & SD \\
\hline Lying & Lying on a sofa & - & - \\
Sitting & Sitting at a computer & 1.23 & 0.16 \\
Light PA & Standing/poster viewing & 1.51 & 0.11 \\
& Table wiping & 2.85 & 0.37 \\
& Floor cleaning & 3.25 & 0.37 \\
Moderate PA & Slow walking & 2.25 & 0.26 \\
Vigorous PA & Fast walking & 5.02 & 0.54 \\
& Soccer & 6.42 & 1.27 \\
& Jogging & 7.53 & 1.05 \\
\hline
\end{tabular}

\section{RESULTS}

Based on the movement patterns of activities, the PAs were classified into five PA classes: lying (lying on a sofa), sitting (sitting at a computer), light PA (standing/poster viewing, table wiping, floor cleaning, and slow walking), moderate PA (fast walking), and vigorous PA (soccer and jogging). METs were used for assessing the intensity of activities.

Total accuracy of the bagged trees prediction model was $96.5 \%$. Specificity and sensitivity for each activity class are presented in Table 4. Mean sensitivity for the model was 95.5\% (SD 3.5) and mean specificity $99.1 \%$ (SD 0.5). For moderate to vigorous PA (MVPA) mean sensitivity was 95.3\% (SD 5.4) and specificity 99.0\% (SD 1.0).

Table 4 Sensitivity and specificity of bagged trees classifier for detecting PA and SED

\begin{tabular}{lcc}
\hline PA & Sensitivity (\%) & Specificity (\%) \\
\hline Lying & 96.4 & 99.2 \\
Sitting & 92.2 & 99.2 \\
Light PA & 98.5 & 99.0 \\
Moderate PA & 91.5 & 99.7 \\
Vigorous PA & 99.0 & 98.3 \\
\hline
\end{tabular}

\section{Discussion ANd Conclusion}

In this study, we developed and validated a method to classify types of PA. The classification was performed using the features extracted from the acceleration signal of the hipworn triaxial accelerometer. In the classification process bagged trees classifier performed with a high accuracy (96.5\%).

Physical activity classification into PA groups according to MET intensity (SED: $\leq 1.5$ METs, light PA: 1.51-2.99 METs, moderate PA: 3-5.99 METs, and vigorous PA: $\geq 6$ METs) is widely used [12, 19-20]. Different thresholds for PA intensity groups has also been used e.g. 4- and 7-MET thresholds to differentiate moderate-intensity PA [21]. In this study, METs were calculated using the standard conversion of $3.5 \mathrm{ml} \mathrm{O} / \mathrm{kg} / \mathrm{min}$, which may not distinguish the individual variability in metabolic responses [7, 22]. Several studies have shown that gender, age and body composition have an impact on energy expenditure of an individual [23-25]. As a consequence, specific thresholds e.g. METs or counts may not be the most optimal for classifying PAs.

A limitation of this study was that participants were adults of working age (17-58 years old). The performance evaluations of proposed algorithm in different populations (e.g. 
children, elderly) will be necessary to conduct before analyzing PA and SED from those populations. Standing still was missing from the measurement protocol of this study, which may also be considered as a limitation. A recent study showed that a hip-worn accelerometer has limited ability to differentiate between sitting and standing postures [11]. The main strength of this study was that classification was performed using ML techniques based on the behavioral characteristics of PAs instead of some specific thresholds. The algorithm developed in this study has a potential for objective evaluations of PA and SED.

\section{ACKNOWLEDGMENT}

The study was supported in part by Polar Electro Oy and the Finnish Funding Agency for Innovation (grant nr 6057/31/2016).

\section{CONFLict of InTEREst}

The authors declare that they have no conflict of interest.

\section{REFERENCES}

1. Lee IM, Shiroma EJ, Lobelo F et al. (2012) Effect of physical inactivity on major non-communicable diseases worldwide: an analysis of burden of disease and life expectancy. Lancet 380(9838):219-229 DOI 10.1016/S0140-6736(12)61031-9

2. Healy GN, Dunstan DW, Salmon J et al. (2008) Breaks in sedentary time: beneficial association with metabolic risk. Diabetes Care 31(4):661-666 DOI 10.2337/dc07-2046

3. Jefferis BJ, Sartini C, Shiroma E et al. (2015) Duration and breaks in sedentary behaviour: accelerometer data from 1566 community-dwelling older men (British Regional Heart Study). Br J Sports Med 49(24):1591-1594 DOI 10.1136/bjsports-2014093514

4. Troiano RP, McClain JJ, Brychta RJ et al. (2014) Evolution of accelerometer methods for physical activity research. Br J Sports Med 48(13):1019-1023 DOI 10.1136/bjsports-2014-093546

5. Leinonen A-M, Ahola R, Kulmala J et al. (2017) Measuring Physical Activity in Free-Living Conditions-Comparison of Three Accelerometry-Based Methods. Front Physiol 7:681 DOI 10.3389/fphys.2016.00681

6. Wijndaele K, Westgate K, Stephens SK et al. (2015) Utilization and Harmonization of Adult Accelerometry Data: Review and Expert Consensus. Med Sci Sports Exerc 47(10):2129-2139 DOI 10.1249/MSS.0000000000000661

7. Welk GJ, McClain J, Ainsworth BE (2012) Protocols for evaluating equivalency of accelerometry-based activity monitors. Med

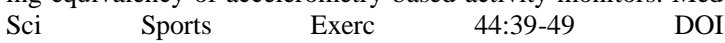
10.1249/MSS.0b013e3182399d8f

8. Vähä-Ypyä H, Vasankari T, Husu P et al. (2015) A universal, accurate intensity-based classification of different physical activities using raw data of accelerometer. Clin Physiol Funct Imaging 35(1):64-70 DOI 10.1111/cpf.12127
9. Zhang S, Rowlands AV, Murray P et al. (2012) Physical activity classification using the GENEA wrist-worn accelerometer. Med $\begin{array}{llll}\text { Sci Sports } & \text { Exerc } & 44(4): 742-748 & \text { DOI }\end{array}$ 10.1249/MSS.0b013e31823bf95c

10. Ellingson LD, Schwabacher IJ, Kim Y et al. (2016) Validity of an Integrative Method for Processing Physical Activity Data. Med $\begin{array}{llll}\text { Sci Sports Exerc 48(8):1629-1638 DOI } & \end{array}$ 10.1249/MSS.0000000000000915

11. Ellis K, Kerr J, Godbole S et al. (2016) Hip and Wrist Accelerometer Algorithms for Free-Living Behavior Classification. Med Sci Sports Exerc. 48(5):933-940.

12. Ainsworth BE, Haskell WL, Whitt MC et al. (2000) Compendium of physical activities: an update of activity codes and MET intensities. Med Sci Sports Exerc 32(9):498-504

13. McLaughlin JE, King GA, Howley ET et al. (2001) Validation of the COSMED K4 b2 portable metabolic system. Int J Sports Med 22(4):280-284 DOI 10.1055/s-2001-13816

14. Antonsson EK, Mann RW (1985) The frequency content of gait. J Biomech 18(1):36-47

15. Zhang S, Murray P, Zillmer R et al. (2012) Activity classification using the GENEA: optimum sampling frequency and number of axes. Med Sci Sports Exerc 44(11):2228-2234 DOI 10.1249/MSS.0b013e31825e19fd

16. Liang Y, Zhou X, Yu Z et al. (2013) Energy-efficient motion related activity recognition on mobile devices for pervasive healthcare. Mobile Netw Appl 19:303-317 DOI 10.1007/s11036-013-0448-9

17. Matthews CE, Hagströmer M, Pober DM et al. (2012) Best practices for using physical activity monitors in population-based research. Med Sci Sports Exerc 44(1):68-76 DOI 10.1249/MSS.0b013e3182399e5b

18. Breiman L (1996) Bagging predictors. Mach Learn 24(2):123140 DOI 10.1007/BF00058655

19. Pate RR, Pratt M, Blair SN et al. (1995) Physical activity and public health: a recommendation from the Centers for Disease Control and Prevention and the American College of Sports Medicine. JAMA 273(5):402-407

20. Gibbs BB, Hergenroeder AL, Katzmarzyk PT et al. (2015) Definition, Measurement, and Health Risks Associated with Sedentary Behavior. Med Sci Sports Exerc 47(6):1295-1300 DOI 10.1249/MSS.0000000000000517

21. Esliger DW, Rowlands AV, Hurst TL (2011) Validation of the GENEA Accelerometer. Med Sci Sports Exerc 43(6):1085-1093 DOI 10.1249/MSS.0b013e31820513be

22. Kozey SL, Lyden K, Howe CA et al. (2010) Accelerometer output and MET values of common physical activities. Med Sci Sports Exerc 42(9):1776-1784 DOI 10.1249/MSS.0b013e3181d479f2

23. Byrne NM, Hills AP, Hunter GR et al. (2005) Metabolic equivalent: one size does not fit all. J Appl Physiol 99(3):1112-1119 DOI 10.1152/japplphysiol.00023.2004

24. Kozey S, Lyden K, Staudenmayer J et al. (2010) Errors in MET estimates of physical activities using $3.5 \mathrm{ml}^{*} \mathrm{~kg}^{-1 *} \mathrm{~min}^{-1}$ as the baseline oxygen consumption. J Phys Act Health 7(4):508-516

25. Melzer K, Heydenreich J, Schutz Y et al. (2016) Metabolic Equivalent in Adolescents, Active Adults and Pregnant Women. Nutrients 8(7):438 DOI 10.3390/nu8070438

Author: $\quad$ Petra Tjurin

Institute: $\quad$ MIPT, University of Oulu

Street: $\quad$ Aapistie 5, P.O. Box 5000

City: FI-90014, Oulu

Country: Finland

Email:_ petra.tjurin @oulu.fi 